\title{
De-mixing of polydisperse fluids: experimental test of a universal relation
}

\author{
D. J. Fairhurst and R. M. L. Evans* \\ Dept. of Physics 8 Astronomy, The University of Edinburgh, U.K. \\ ${ }^{*}$ To whom correspondence should be sent at current address: \\ Dept. of Physics \& Astronomy, University of Leeds, LS2 9JT, U.K. \\ E-mail: mike.evans@physics.org
}

31 March 2003

\begin{abstract}
Colloids, emulsions, polymer blends, and other important complex fluids, are 'polydisperse', i.e. there are variations among their constituent particles. Polydispersity is usually regarded as a ubiquitous, uncontrollable nuisance causing experimental inconsistencies. We have varied the polydispersity of a complex fluid, whilst keeping all other parameters constant, and report the first measurements of some universal physics. At coexistence (e.g. between liquid and vapour), fractionation occurs - each phase receives a different mix of the various ingredients e.g. with the liquid disproportionately abundant with larger particles. Theory predicts, at low polydispersity, that this de-mixing becomes universal, irrespective of the material, with chemical differences between the phases proportional to polydispersity to the power 2 . We study colloid-polymer suspensions at two-phase coexistence and, using light scattering, measure the exponent as $2.16 \pm 0.44$.
\end{abstract}

Key words: polydispersity, fractionation, coexistence, universality.

\section{Introduction}

Simple fluids are composed of identical molecules, but many currently important fluids are elaborate mixtures of disparate particles. Crude oil, for example, contains thousands of chemical components, while in colloids, no two particles are strictly identical, no matter how much care is taken to achieve consistency in their production. The properties of complex mixtures are proportionately diverse. When two phases coexist (e.g. liquid oil and its vapour, or milk and a separated layer of cream), each phase receives a different mix of the various ingredients - they fractionate. Predicting the constituent proportions usually requires a detailed knowledge of the particular system. However, de-mixing behaviour is expected $[1,2,3]$ to become universal if there is sufficiently little variation between particles. A recent theory $[1,2,3]$ predicts that the difference in mean particle radii $\Delta\langle r\rangle$ 
(in the case of size polydispersity, e.g. for emulsion droplets) between coexisting phases, scales with the overall mean $\langle r\rangle_{o}$, and with the square of polydispersity $\sigma$, in the limit of small $\sigma$, i.e.

$$
\Delta\langle r\rangle=\gamma\langle r\rangle_{o} \sigma^{2}
$$

The coefficient $\gamma$ is non-universal. It depends on intensive properties of the two phases, but not on the amounts of the phases, so that it does not vary along a tie line. Specifically, the derivative of the free energy per particle with respect to mean particle size, at constant number density, is different for the two phases. (That is why it is advantageous for one phase to expel large particles into the other.) This difference between the free energy derivatives in the two phases, expressed in dimensionless units, defines $\gamma[1,2]$. Because the radii of emulsion droplets vary, the polydispersity $\sigma$ is defined here as the standard deviation in the radii divided by the mean radius, but it can be defined in terms of molecular weights, aromaticity, or whatever property distinguishes the constituents of the substance in question. The same relation (Eq. 1) is expected always to hold at low $\sigma$.

That is only a theoretical prediction but, if true, it obviously has wide-ranging implications, as experiments are generally performed on low- $\sigma$ materials, in attempting to attain the monodisperse ideal $(\sigma=0)$. Despite its ubiquity, the effects of polydispersity remain obscure, since it turns up, unbidden, at a fixed (often unknown) value that cannot easily be independently varied. To experimentally test the theoretical universal relation (Eq. 1) is therefore a far from trivial task. It requires very precise synthesis and measurement of mixtures with small, but different, polydispersities. Meanwhile, in order to hold $\gamma$ fixed in Eq. 1, all other conditions in the mixtures must be held constant, i.e. the differently synthesized samples must be taken to identical states. In practice, it is most important for conditions in the denser phase to remain constant, as this is where the free energy is most sensitive to variations in particle size.

\section{Experimental test}

The experimental system used as a test bed was a mixture of poly-methylmethacrylate (PMMA or 'Perspex') colloidal spheres with random coils of polystyrene, suspended in cis-decahydronapthalene. The PMMA particles were coated with a short, brush-like layer of poly-hydroxystearic acid to prevent aggregation. Their synthesis is described in the literature $[4,5]$. For near-monodisperse colloid, the phase diagram of this system has been well charted [6, 7]. It is the archetypal system of attractive hard spheres. At high colloidal concentration $\phi$, the spheres are efficiently packed in a crystalline arrangement, while amorphous phases exist at lower concentrations. If the size ratio $\xi$ of polymer coils to colloidal spheres is greater than about 0.3 , the system is known to exhibit an additional transition between amorphous phases at intermediate and low $\phi$, known respectively as "colloidal liquid" and "colloidal gas". For a given size ratio $\xi$, the phase diagram is independent of the absolute particle sizes. The established $[6,7]$ phase diagram for nearmonodisperse mixtures with $\xi=0.45$ is shown in Fig. 3. Henceforth, we choose to concentrate on the region of gas-liquid equilibrium coexistence, since separation of a polydisperse crystalline phase is beset with issues of non-ergodicity [8].

The aim of this study is to examine how the partitioning of colloidal particle sizes 
between two phases depends on the polydispersity. Our approach was to prepare samples of various $\sigma$ in a state that de-mixes into co-existing phases. To experimentally determine the effect of polydispersity alone, all other quantities must be held fixed, which means taking care to prepare samples at equivalent points in the phase diagram, although they are taken from different stock solutions. Three stock solutions of colloidal PMMA were used, with polydispersities $\sigma=(5.2 \pm 0.6) \%,(7.4 \pm 0.4) \%$ and $(18.1 \pm 0.6) \%$ and mean radii of 244,300 and $170 \mathrm{~nm}$ respectively. As these mean radii differ, a separate polymer solution was prepared for each colloidal stock, to ensure a constant size ratio of $\xi=$ $0.45 \pm 0.03$.

To eliminate systematic errors, three mixtures were prepared from each stock, at fractionally different, but almost identical, compositions. Each of the nine samples was thoroughly mixed and then left undisturbed to separate into two phases. In all cases, a faint boundary emerged and gradually sharpened, dividing a concentrated colloidal liquid from dilute colloidal gas, as in Fig. 3(inset). After approximately two days, when the only observable change was slow settling under gravity, the separation was presumed to be complete. The initial compositions inside the gas-liquid coexistence region are shown in Fig. 3, as are pairs of points on the phase boundaries, representing final states of twophase equilibrium. (Coexisting pairs can be identified in the figure, since they always lie on a straight line that passes through the initial composition. Some of the tie lines are shown in the figure.) We see that the final phases are in two groups of rather similar compositions (all the colloidal gases are at low colloidal concentration and high polymer concentration, while the colloidal liquids are high in colloid, low in polymer). The scatter of compositions in each of the two groups has no systematic variation with $\sigma$. We have thus achieved our goal of visiting the same part of the phase diagram for samples of different $\sigma$.

After separation, representative samples from each pair of phases were manually removed using a syringe, and highly diluted. Mean particle radii in these samples were determined by light scattering (see Fig. 2). The diluted samples were illuminated with a krypton ion $\left(\mathrm{Kr}^{+}\right)$laser, and the scattered (diffracted) light intensity measured as a function of scattering angle (shown as a polar plot in Fig. 2), yielding the form factor of the suspended colloidal spheres, the polymer coils being effectively invisible. The form factor was fitted to a known analytic form [9], blurred only a little by variation in particle size, since all polydispersities were small. Thus the mean particle size $\langle r\rangle$ in each phase was extracted (basically given by the angles of the minima in Fig. 2), and its difference $\Delta\langle r\rangle$ between coexisting phases, scaled by the initial mean $\langle r\rangle_{o}$ in the colloidal stock, is plotted in Fig. 3 against the stock polydispersity $\sigma$.

\section{Results}

The data were fitted by a power law (a straight line in the log-log plot). The least-squares fit was weighted by inverse-square uncertainties [10]. The error bars (one standard deviation) in Fig. 3 are sizeable since the data are obtained from the difference between two very similar measurements ( $\langle r\rangle$ in each phase). The scatter of data from each colloidal stock (at fixed $\sigma$ ) is of similar magnitude to the uncertainties in light-scattering measurements 
for each sample, confirming that systematic errors due to variations in phase-diagram position are negligible. The uncertainties in the data were propagated to find the uncertainty (one standard deviation, $68 \%$ confidence level) in the gradient. The exponent (gradient in the log-log plot) was determined to be $2.16 \pm 0.44$, in good agreement $[1,2]$ with the theoretical value of 2 .

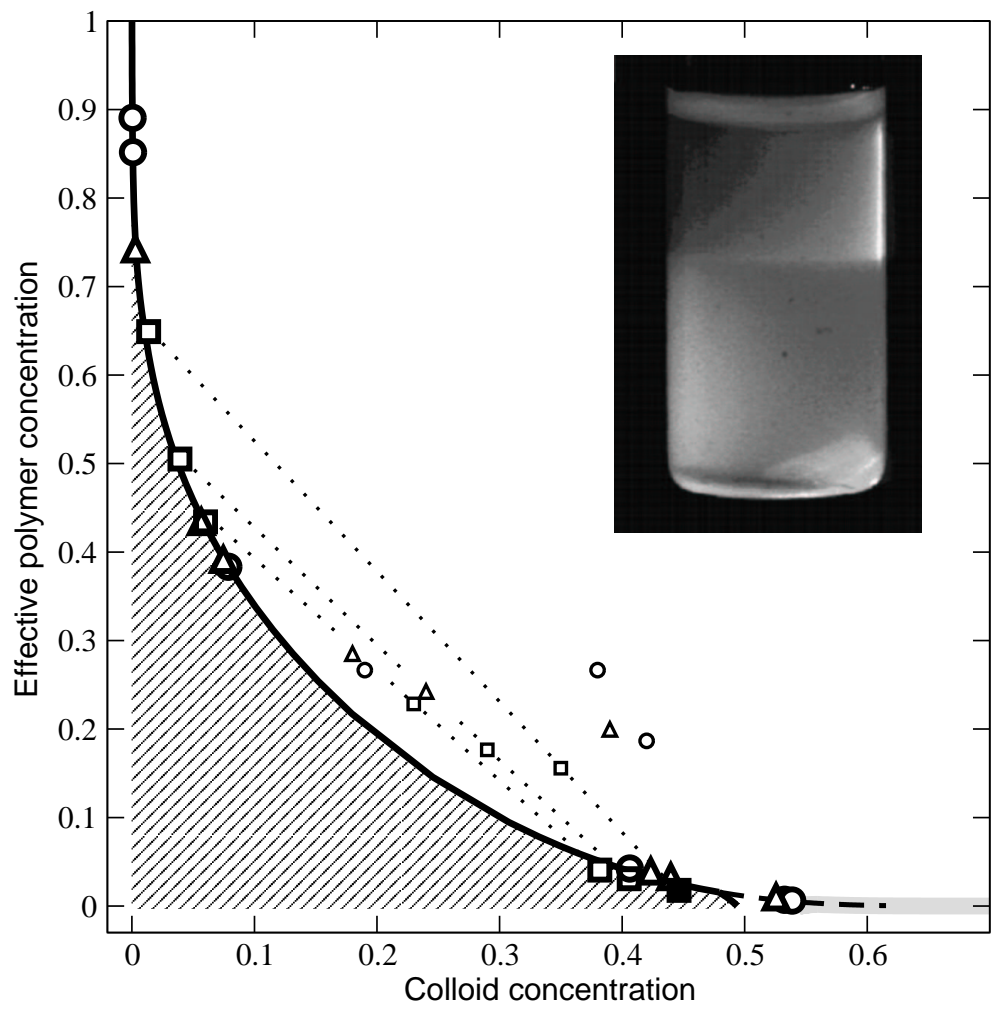

Figure 1: The established monodisperse phase diagram $[6,7]$ for $\xi=0.45$, in the plane of polymer concentration (relative to that at which polymer coils overlap) vs. colloidal volume fraction $\phi$. Colloidal gas, liquid or fluid phases (hatched), crystalline phase (grey) and coexistence regions (white) are shown. The dashed curve is a metastable continuation of the colloidal liquid-gas coexistence line, that becomes stable for polydisperse samples in which crystallization is suppressed. Initial (small symbols) and final (large symbols) locations of all nine samples are indicated for polydispersity $\sigma=5 \% \triangle, 7 \% \bigcirc, 18 \% \square$. Tie lines (dotted) linking the initial and final states are shown for the $\sigma=18 \%$ samples. Data for the colloidal liquid sample highlighted (filled black square) are shown in Fig. 2. Inset: A sample at colloidal gas-liquid coexistence. An interface between the two phases is clearly visible across the middle of the sample tube.

\section{Conclusion}

Unlike in theoretical and computational approximations $[11,12,13,14,15,16]$, polydispersity $\sigma$ is inherent, and often uncontrollable in the real world. In experiments, it is not easy to alter the polydispersity whilst holding other parameters fixed. Indeed, in a previous experiment [1] on the low- $\sigma$ colloid-polymer mixtures, where the shapes of the 


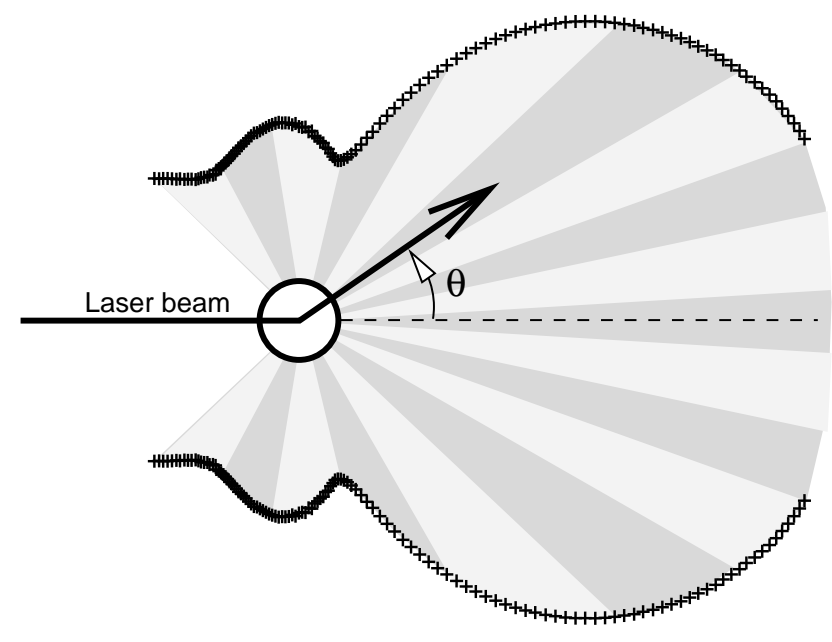

Figure 2: Light scattering geometry. The measured intensity of light scattered from the diluted sample (carrying information on particle sizes), is shown logarithmically in the polar plot as a function of scattering angle $\theta$, for the sample indicated by a filled square symbol in Fig. 3.

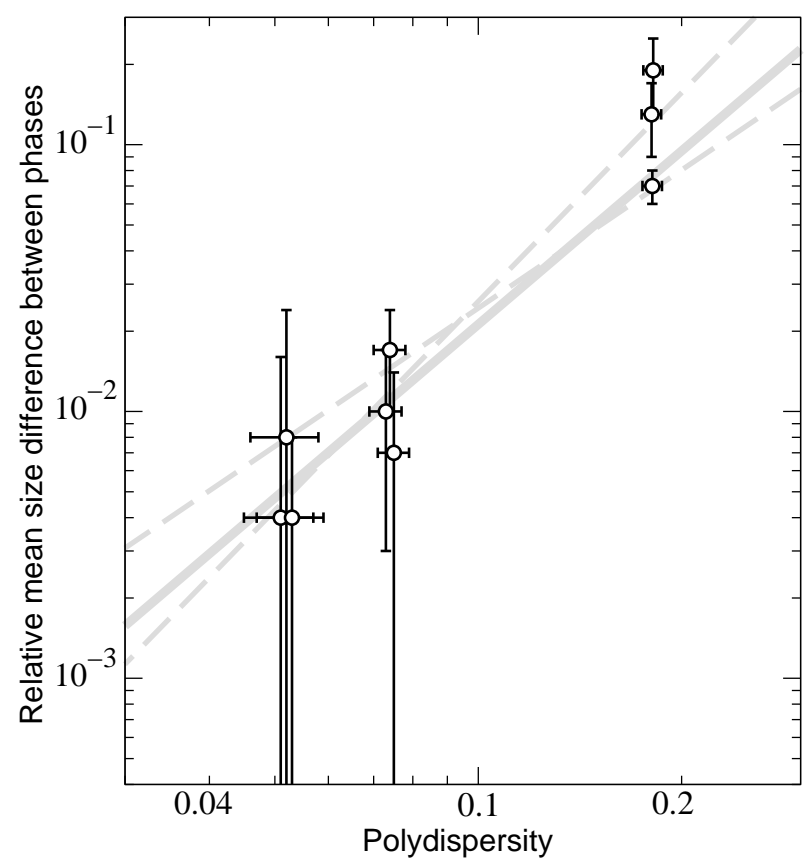

Figure 3: Log-log plot of fractional difference in mean radii of particles in coexisting phases, $\Delta\langle r\rangle /\langle r\rangle_{o}$, versus initial polydispersity $\sigma$. Note error bars indicating not all data have equal weights. The solid line shows the best-fit (weighted by inverse-square uncertainties[10]) power law with exponent $\mu=2.16$. Dashed lines have gradients $\mu \pm \delta_{\mu}$ indicating the uncertainty in the exponent at the level of one standard deviation $(68 \%$ confidence), $\delta_{\mu}=0.44$. 
size distributions in coexisting phases were found to agree with predictions, all samples were taken from a single stock of colloid to facilitate calibration, so the initial polydispersity could not be varied. In the present study, we havesucceeded in varying $\sigma$ across a set of experimental samples, whilst identically reproducing all other conditions. This involved replicating the details of the microscopic interactions as well as keeping the concentrations of colloid and polymer constant in the coexisting phases. The measurements become particularly difficult for the small values of polydispersity at which de-mixing becomes universal. For this reason, the simple power-law behaviour we have observed, though expected to be commonplace, has not previously been accessed unambiguously in the laboratory.

Acknowledgements Thanks go to Suzanne Fielding for helpful suggestions. RMLE is a Royal Society University Research Fellow.

\section{References}

[1] Evans RML, Fairhurst DJ, Poon WCK (1998) Phys Rev Lett 81: 1326

[2] Evans RML (2001) J Chem Phys 114: 1915

[3] Fredrickson GH (1998) Nature 395: 323

[4] Antl L, Goodwin JW, Hill RD, Ottewill RH, Owens SM, Papworth S (1986) Colloids and Surfaces 17: 67

[5] Pathmamanoharan C, Slob C, Lekkerkerker HNW (1989) Colloid Polymer Sci 267: 448

[6] Ilett SM, Orrock A, Poon WCK, Pusey PN (1995) Phys Rev E 51: 1344

[7] Lekkerkerker HNW, Poon WCK, Pusey PN, Stroobants A, Warren PB (1992) Europhys Lett 20: 559

[8] Evans RML, Holmes CB (2001) Phys Rev E 64: 011404

[9] Pusey PN, van Megen W (1984) J Chem Phys 80: 3513

[10] Taylor JR (1997) An introduction to error analysis. University Science Books, Sausalito California

[11] Barrat JL, Hansen J-P (1986) J Physique 47: 1547

[12] Clarke N, Cuesta JA, Sear R, Sollich P, Speranza A (2000) J Chem Phys 113: 5817

[13] Bartlett P, Warren PB (1999) Phys Rev Lett 82: 1979

[14] Sollich P, Warren PB, Cates ME (2001) Adv Chem Phys 116: 265

[15] Pagonabarraga I, Cates ME, Ackland GJ (2000) Phys Rev Lett 84: 911

[16] Kofke DA, Bolhuis PG (1999) Phys Rev E 59: 618 